COLLOQUE DE PHYSIQUE

Colloque C2, supplément au $\mathrm{n}^{\circ} 2$, Tome 51, Février 1990

ler Congrès Français d'Acoustique 1990

\title{
MESURES D'IMPÉDANCES, DE FONCTIONS DE RÉFLEXION ET D'HARMONICITÉ D'INSTRUMENTS À VENT
}

\author{
V. GIBIAT et F. LALOE* \\ Groupe de Physique des Solides, CNRS UA 17, Université Paris 7 T23, 2 \\ Place Jussieu, F-75251 Paris Cedex 05. France \\ * Laboratoire de Spectroscopie Hertzienne de I'E.N.S., CNRS UA 18, 24 \\ Rue Ihomond, F-75005 Paris, France
}

\begin{abstract}
Résumé - La méthode de mesure de l'impédance d'entrée des instruments à vent dite T.M.T.C. que nous avons développé récemment permet grâce à deux mesures simultanées de pression et à une calibration en trois points d'obtenir l'impédance d'entrée d'instruments à vent de diamètres variés avec un seul dispositif de mesure dont les imperfections géométriques acoustiques et électriques sont automatiquement prises en compte. L'utilisation systématique de la micro-informatique et de la saisie numérique permet de disposer d'un outil souple et rapide. Il est ainsi possible de calculer à partir de la mesure d'impédance la fonction de réflexion de l'instrument étudié ainsi que toute autre fonction temporelle. La mesure précise des positions des maximums d'impédance peut également être interprêtée en fonction de leur rang et donner une indication sur l'harmonicité ou l'inharmonicité des résonnances de l'instrument et donc sur sa qualité.

Abstract - We have developped recently the T.M.T.C method in order to measure the input impedance of woodwinds. This method allows us with a two pressure measurement and a three calibration technic to obtain the impedance of various diameter instruments and to correct a lot of systematics such as geometrical imperfections, microphone admittance,.... The intensive use of micro-computing and numerical acquisition gives us a powerful and fast tool. We are able to compute from the impedance the reflection function or any other time domain function. The knowledge of the frequency position of the impedance peak gives an information of the quality of an instrument by drawing the harmonicity or inharmonicity curve of the instrument.
\end{abstract}

\section{1- INTRODUCTION}

Les méthodes de mesure de l'impédance d'entrée des instruments à vent sont nombreuses et souvent très satisfaisantes, néanmoins il n'existe pàs à notre connaissance de système à haut niveau d'exitation (permettant de s'affranchir de l'utilisation d'une chambre sourde) rapide (temps de mesure de l'ordre d'une dizaine de secondes) et suffisament précis à la fois en fréquence et en amplitude. Les systèmes classiques à deux microphones /1,2/couplés à une informatique légère sont de bons candidats (fig. 1) mais il n'est pas possible de les utiliser avec des diamètres différents et les réalisations orientées vers la mesure de l'impédance de matériaux ne possédent pas le haut degré de résolution dynamique indispensable à la mesure sur des instruments à vent et négligent le plus souvent pertes visco-thermiques et erreurs liées à la mesure telle que l'admittance des microphones et leur influence sur la géométrie de la cavité. Nous avons donc développé une méthode de mesure indépendante de la géométrie de la cavité de mesure et tenant compte des pertes.

\section{2 - LA METHODE TMTC}

La figure 2 donne le principe de la mesure. L'idée générale pour une exitation monochromatique revient à écrire en supposant une propagation en onde plane dans la région du plan $x=0$ les signaux acoustiques $s_{1}$ et $s_{2}$ mesurés par les deux microphones $M_{1}$ et $M_{2}$ en fonction de $p$ et $v$ pression et débit dans le plan $x=0$. 


$$
\begin{aligned}
& s_{1}=\alpha p+\beta \rho c v \\
& s_{2}=\gamma p+\delta \rho c v
\end{aligned}
$$

avec $\alpha, \beta, \gamma, \delta$ des paramètres dépendant de la géométrie du système.

En posant $Z=p / v$ et $y=s_{2} / s_{1}$ on obtient

$$
Z=\rho c \frac{-\beta y+\delta}{\alpha y-\gamma}=\rho c \frac{A y+B}{y-y_{0}}
$$

où les coefficients $A, B, y_{0}$ sont les coefficients à déterminer pour calibrer le dispositif. Il suffit de mesurer y pour trois impédances connues pour déterminer ces coefficients. Les trois coefficients connus pour l'ensemble des fréquences il suffit de mesurer $y$ pour l'impédance inconnue pour obtenir $Z$. Les impédances de référence utilisées sont deux cavités cylindriques fermées dont l'impédance théorique est parfaitement connue pertes y comprises et une terminaison d'impédance infinie qui donne directement $y_{0}$.

Pour une géométrie du dispositif de mesure parfaitement cylindrique les coefficients $A, B, y_{0}$ se réduisent aux valeurs theoriques et pratiques classiques $/ 2 \%$.

Cette méthode de calibration en trois points permet d'améliorer les performances d'un système à deux microphones et elle est indispensable pour mesurer l'impédance de cavités de diamètres différents de celui de la cavité de calibration. Des contingences de précision de mesure conduisent à coupler la cavité de mesure et le système acoustique à étudier à l'aide d'un petit cône. La figure 3 présente les résultats obtenus pour trois cavités de diamètres différents. Les résultats sont comparables et aussi bons pour les pics que les creux ceci sans nécessiter de chambre sourde.

L'ensemble du dispositif de mesure est géré par un micro-ordinateur de type AT equipé d'une carte $\mathrm{N} / \mathrm{A}$ et $\mathrm{A} / \mathrm{N}$ qui génère le signal d'exitation (du type "chirp") sur .5 secondes et acquiert simultanément les signaux électriques $s_{1}$ et $s_{2}$ sur 1 seconde. Pour une meilleure précision nous utilisons trois voire quatre microphones. Le couple le plus écarté étant réservé aux basses fréquences. Dès lors que l'impédance est calculée et stockée dans une mémoire de masse il est très simple d'obtenir la fonction de réflexion et la courbe d'harmonicité de la cavité en portant la valeur des fréquences de résonnances en fonction du rang des pics.

\section{3 - MESURES SUR DES INSTRUMENTS A VENT.}

Nous avons étudié plus particulièrement deux instruments de la famille des clarinettes. Un certain nombre de clarinettes en SIb pour laquelle notre dispositif est plus particulièrement adapté et une clarinette basse. La figure 4 a été obtenue pour une clarinette Sib pour la note la plus grave avec sans clé de registre. la figure 5 concerne le Sol clairon d'un clarinette basse. Les notions de fréquence de coupure et l'influence de la clé de registre sont très visibles et on peut remarquer sur la figure 5 la réapparition de pics d'impédance pouvant être liés à une seconde bande passante.

La figure 6 présente les fonctions de réflexions correspondantes.

\section{4 - COURBES D'HARMONICITE.}

Le fonctionnement correct des instruments à vent est lié à la plus ou moins bonne correspondance entre les fréquences des résonnances (ici les maximums d'impédance) et les fréquences d'une série harmonique/3/. Nous avons porté sur un même graphique (fig. 7) les courbes obtenues pour un certain nombre de doigtés d'un même instrument. L'écart entre les points de mesure et la droite d'harmonicité idéale permet d'avoir une idée de la facilité d'émission des notes concernées. Il est aisé de voir que le mi grave sera d'autant plus bas que le nombre de pics concernés sera grand (cas d'un jeu forte) alors que le sol est pour cet instrument très harmonique. 


\section{5 - CONCLUSION.}

La méthode de mesures d'impédance que nous présentons ici est clairement reliée à la méthode du tube de Kundt qui a récemment été reprise et améliorée pour l'impédance des matériaux /2/. Le principal intérêt de notre procédure de calibration est de permettre la mesure sur des instruments à vent de diamètres variés. Elle permet également une compensation automatique des erreurs qui entâchent souvent ce type de méthode et de ce fait évite l'emploi de microphones de mesure. Son implémentation sur un microordinateur standard et l'emploi de matériel de faible coût permet d'envisager sa diffusion hors des laboratoires spécialisés. Enfin la rapidité de l'acquisition (quelques secondes) et de traitement (quelques minutes) en font un outil particulièrement souple.

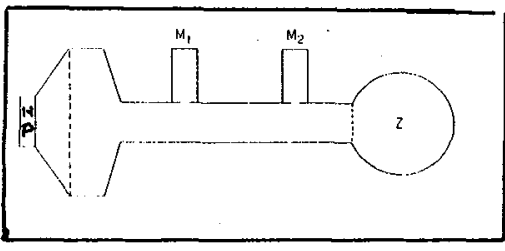

figure 1 :

Méthode à deux microphones.

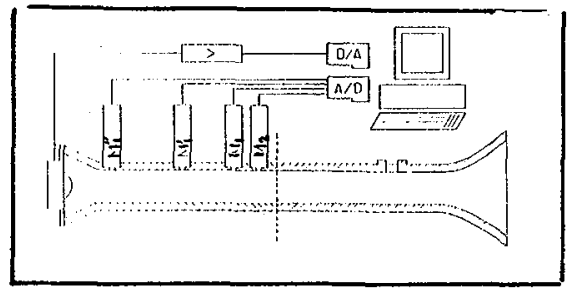

figure 2 :

Méthode TMTC.
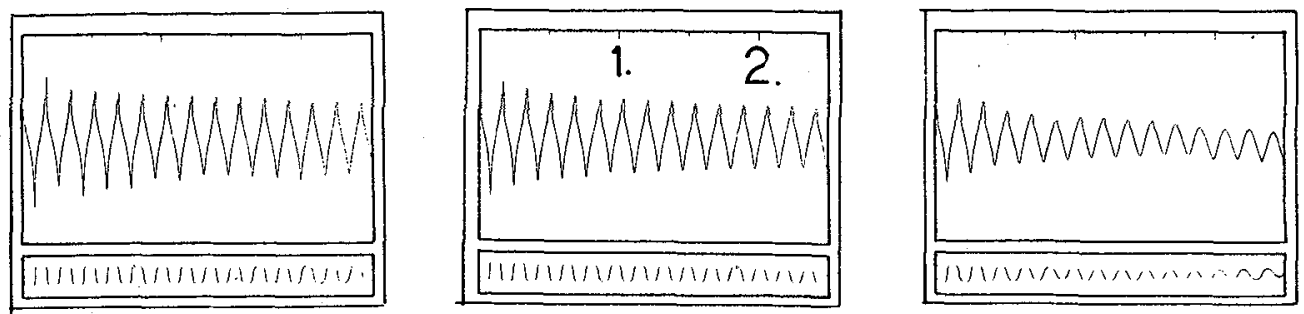

figure 3 :

Impédance de trois cavités cylindriques de longueur $1 \mathrm{~m}$ et de diamètres 24, 15 et $6 \mathrm{~mm}$. (Echelle Log.).
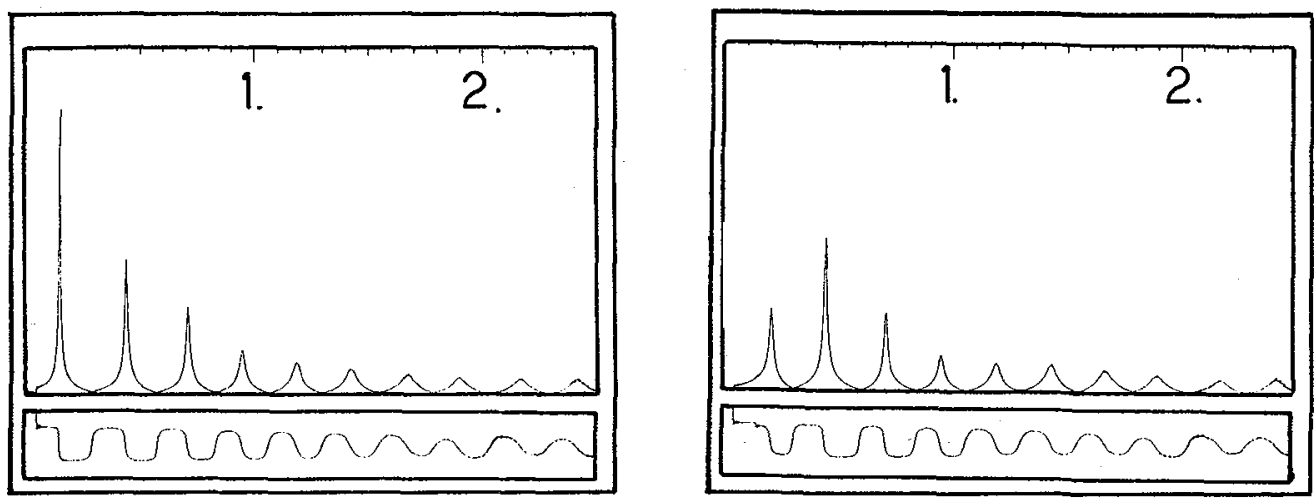

figure 4 :

Impédance du mi grave et du si (même doigté plus clé de registre)

d'une clarinette sib. 


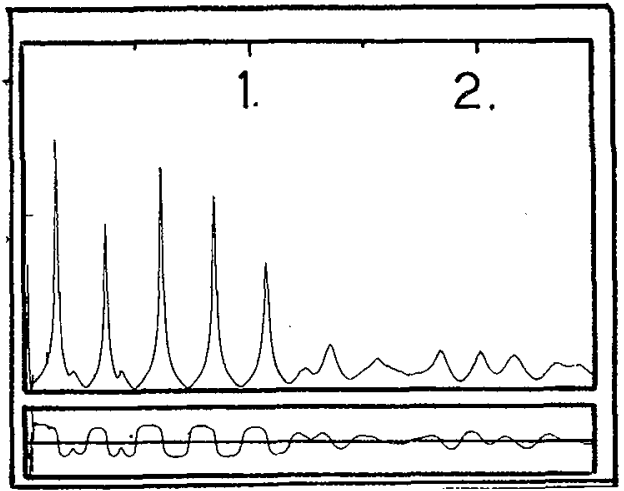

figure 5 :

Impédance du sol clairon d'une clarinette basse.
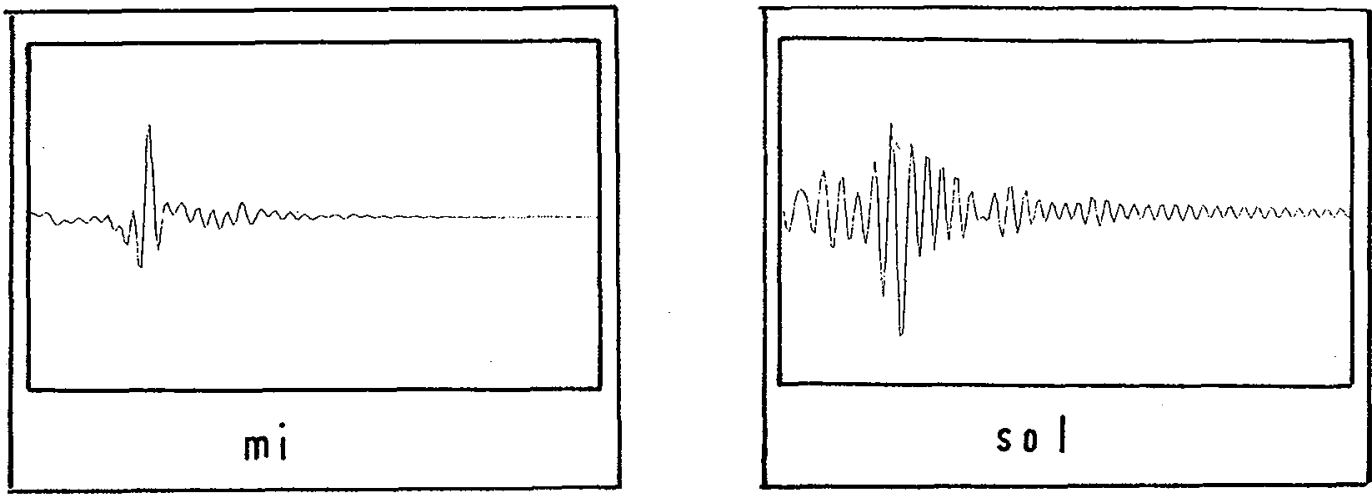

figure 6 :

Fonctions de réflexion correspondant aux figures 4 et 5 .

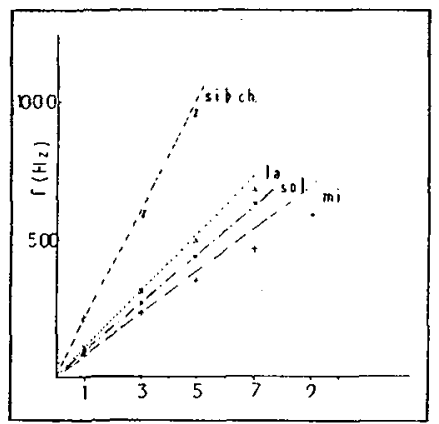

figure 7 :

courbes d'harmonicité de différents doigtés d'une clarinette Sib.

\section{REFERENCES}

/1/ Desmet B. et Maton M., C. R. Acad. Sc. B280,(1975) 333.

/2/ Chung J.Y. et Blaser D.A. , J. Acoust. Soc. Am. 62, (1980)

/3/ Benade A.H. Fundamentals of musical acoustics ,Oxford University Press (1976). 